\title{
THE RELATIONSHIP BETWEEN BIORHYTHM (PHYSICAL CYCLE) AND SPORTS PERFORMANCE IN WOMEN'S BASKETBALL
}

\author{
Shafiee Shahram ${ }^{1}$, Rahim Ramezaninezhad ${ }^{1}$, Hakime Afrouzeh ${ }^{1}$, Vahid Rabbani ${ }^{2}$ \\ ${ }^{1}$ University of Guilan, Iran \\ ${ }^{2}$ Allameh Tabataba'i University, Tehran, Iran
}

\begin{abstract}
Purpose: Despite many researches that have been done in the field of biorhythm and due to lack of single view among scholars, as well as importance of forecasting of athletes' performance to improve their results, the purpose of this study is to determine relationship between biorhythm (physical cycle) and sports performance of Iranian Super League women basketball players. Material: This is a descriptive-correlational study. Statistical population was women basketball players in 1394-95 women's Super League and the statistical sample included all of the players of the teams, qualified as semi-finalists (44 players). The tool, used in this research, was athletes' performance questionnaire (Charbonneau, 2001) containing five questions by a Likert scale from 1 (poor) to 5 (excellent) points. Biorhythm software and descriptive tests and also inferential statistical test including ChiSquare, independent T-test and one way ANOVA at significance level of $(\alpha \leq 0.05)$ in SPSS software were used for analyzing the collected data. Results: results showed that there was no significant relationship between physical energy and performance of athletes $(\mathrm{p}=0.85)$. Also the results of one way ANOVA test showed that there was no significant difference between performance of athletes at three levels (positive, negative and critical) and physical cycle $(p=0.96)$. The value of Chi-Square was equal to 2.63 that showed there was no significant relationship between different levels of physical cycle and the results (win or lose) of match $(p=0.026)$. Conclusion: The results of this study revealed that physical performance did not depend on 23-day cycle, stated in the Biorhythm theory, and there was no evidence proving existence of these cycles. It can be concluded that there is no justifying reason to use the Biorhythm software in hard exercises.
\end{abstract}

Keywords: biorhythm, performance, sports, physical cycle, women's sport.

\section{Introduction}

Biorhythm is one of the newest topics in the area of mind ergonomics identification, which can be very effective in reducing work related accidents and any mistakes without apparent reasons through identifying intellectual, physical and emotional aspects of a person [4, 7]. Today, there is too much emphasis on attending different aspects of human resources, both physical and non-physical in order to maximize individual and organizational productivity and the prerequisite for this is identifying human resources accurately in terms of physical and intellectual ergonomics. One of the most essential elements in this kind of identification is using biorhythm, which represents their intellectual, physical and emotional characteristics in different days of their lives $[10,9]$.

The changes in stamina and ability to carry out everyday activities, such as exercising and participating in a party, feeling healthy or sick, dealing with problems and even intellectual order or critical moments of life that an individual experiencing his/her worst or best conditions in terms of energy are categorized in three alternating cycles, namely physical, emotional and intellectual, which altogether form the individuals ' biorhythm [13]. Awareness about the situation of physical, emotional and intellectual energy can help increase individual's productivity. For example, when the physical energy is in its peak, it is the best time for doing heavy works, sports, surgical practices and making vital decisions. In the course of positive thinking and perception, one must act more carefully and avoid risky activities, because there is a high possibility of error and making mistakes in this period $[21]$.

The word "Biorhythm" has been derived from the Greek words "bios", meaning life, and "rhythmus", meaning regular and thoughtful move. From the perspective of Biorhythm theory, humans are being influenced by physical, emotional and intellectual cycles from the day they are born and continuing until death $[19,6]$. These cycles include 23-day physical cycle, 28-day emotional cycle and 33-day cognitive cycle, each of which has the following features $[11,5]$ :

a. A high-level or positive phase;

b. A low-level or negative phase;

c. A critical day.

\footnotetext{
(c) Shafiee Shahram, Rahim Ramezaninezhad,

Hakime Afrouzeh, Vahid Rabbani, 2016

doi:10.15561/20755279.2016.0308
} 


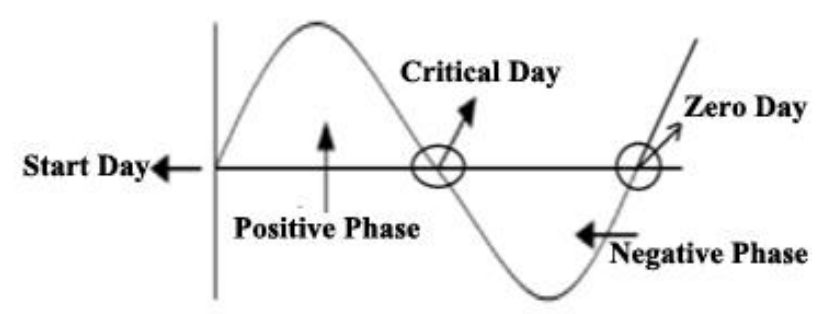

Fig. 1. Different parts of biorhythm cycle.

As it is seen in figure 1, the top part of the curve shows the positive phase. A positive phase includes those days when the body releases its energy and is likely to be at its peak. The lower part of the curve shows the negative phase in which the body returns the released energy in the positive phase and is likely to have a below normal performance. The number of days in this phase is equal to the number of days in positive phase. The body tends to instability on critical days. The critical point is an almost neutral point belonging to one of the positive or negative cycles or it does not belong to any of them at all [11].

It seems that half of the time period of each cycle is positive and the other half is negative. In other words, in each first 11.5 days, physical activity is associated with mobility, energy, high physical strength, accurate and effective work together with endurance and stability. Weakness, fatigue, lack of stored energy and lack of ability are observed in the next 11.5 days. Optimism, willingness to work and live and active participation in works are observed in the first 14 days of emotional cycle. The second 14 days are associated with bad temper, irritability, impatience and moroseness. Similarly, for intellectual cycle, after the first 16.5 days, intelligence, understanding, high analytical spirit and creativity will be replaced by second 16.5 days and its features such as forgetfulness, laziness of thought and difficulty in focusing and decision making [8, 12].

\section{Physical cycle}

Phyllis believed that physical cycles originate from muscle tissues. This cycle refers to the masculinity of people and affects their physical conditions. It is believed that there is a concurrence between adrenal gland and physical cycle in the body. This cycle continues for 23 days and associates with strength, mobility, determination, endurance and innovation. When the physical cycle is above the baseline ( $2^{\text {nd }}$ to $11^{\text {th }}$ day), the physical condition of person is charged and will be discharged gradually. In such a situation, the person is able to do better in physical works and feels stronger and more enthusiastic than before. There is a low probability of the person becoming ill and he/she can also endure and handle more pressure. Some doctors believe that between the second and ninth days, when the patient's physical cycle is well placed in positive area, is the best time to do surgery. On the other hand, when the physical cycle is placed below the baseline $\left(13^{\text {th }}\right.$ to $23^{\text {rd }}$ day), the released energy will be charged gradually. In such situations, the person simply gets tired and is susceptible to cold and other diseases $[2,3,15 \&$ $13]$.

\section{Emotional cycle}

This cycle dominates in nervous system. It is more related to femininity of a person and affects a person's feelings. In fact, all women feel the existence of this cycle in their bodies. This is a 28-day cycle which controls sensitivity, emotional issues, temperament, moods, nervous system and creativity. As long as this cycle remains above the baseline ( $2^{\text {nd }}$ to $4^{\text {th }}$ days), individual's creativity, emotions, love and spirit of cooperation is in a good condition and he/she is more optimistic and happier than before. On the contrary, people feel bored, grumpy and depressed when this cycle is placed below the baseline (the $16^{\text {th }}$ to $28^{\text {th }}$ days). Also people become more cantankerous and irritable than before in such situations $[1,4]$.

\section{Perceptual or Intellectual cycle}

This cycle continues for 33 days and affects individual`s learning ability, analytical thinking, reasoning, judgment and decision-making. When this cycle is placed above the baseline ( $2^{\text {nd }}$ to $16^{\text {th }}$ days) people are able to think more intelligently, solve the problems more effectively, perform more successfully in the exams and make decisions more accurately. This is an appropriate time for creative thinking and producing novel ideas. But when the cycle is placed below the baseline ( $18^{\text {th }}$ to $33^{\text {rd }}$ days), individual's thinking capacity is reduced. They show a poor judgment and memory performance falls off. They may find it hard to focus or may take wrong decisions. This period may be a good time to review previous known ideas (Moemeni Piri et al, 2012). Biological foundation of some of body cycles is completely recognized. The most well-known cycle is the menstrual cycle of women 
which follows a certain time rhythm and regularly repeats every 28-35 days. The researchers believe that even the most complex human functions such as intelligence, emotions and physical skills follow such cycles that start from the birth and are repeated regularly throughout the life [9].

Rabiei and Khatami No (2011) showed a significant relationship between biorhythm and job satisfaction. Also Hosseini and Mahdi Zadeh (2009) indicated in their study that there is a significant relationship between biorhythm and academic performance.

A handful of studies have been done about Biorhythm theory in sports some of which confirmed the theory while some showed inconsistency in their results. In a research conducted by Faria and Elliott (1980), the maximum consumed oxygen of vo2max by female gymnasts in the critical days of physical biorhythm cycle was tested and the result showed that physical biorhythm cycle does not have a significant effect on aerobic capacity of athletes. Maura (2009) in study examined the relationship between the biorhythm cycles and sports performance of 61 students from four teams in three phases in football final matches and the results showed a significant relationship between performance and biorhythm cycles.

Shaebani Bahar et al. (2013) examined the relationship between competition results and physical, intellectual and emotional cycles in a student's Olympiad. They concluded that there is a significant relationship between physical, intellectual and emotional cycles with the competition results. Moldovan et al. (2011) studied the performance of gymnasts and biorhythm and the results showed that gymnasts had a better performance in positive phase. Souatra and Wiyor (2002) in a 15-month study showed that people`s critical days of biorhythm has been the primary cause of work-related accidents. Taylor's research (2004) on employees of the UK electricity company revealed that there is a significant relationship between biorhythm and reduction of work-related accidents and that there is a significant relationship between more accurate decisions of senior managers and their good and bad days. Willey \& Farnkoni (2003) mentioned lack of attention to biorhythm of people, especially its emotional aspect, as the primary cause of reduction in efficiency or employees quality of work in administrative jobs.

In fact, the purpose of studying biorhythm cycles or identifying and calculating people`s rhythmic cycles is to determine the impact of these rhythms on individual's conditions, calculate the optimal and critical days, perform activities optimally, predict weakness and strength times and not to perform some activities in critical days (Dehghan, 2008). Researchers believe that knowing about the athletes' positive and negative phase or critical days of physical cycle by the coach or other people, who are in contact with them, can increase productivity, prevent sports injuries, improve performance and other factors influencing physical performance of athletes (Zareian et al., 2014). However, there are very limited and conflicting results about the application of Biorhythm theory in sports. Some supporters of Biorhythm theory refer to the relationship between success of athletes and the days when their biorhythm is in a positive condition and others believe that there is no relationship between biorhythm and athletes' success. Lack of research in this field either overseas or local has induced researchers to try to answer the following questions: Is there any relationship between the performance of athletes and their biorhythm?

Hypothesis: This study tries to evaluate the relationship between physical dimension of biorhythm and performance of the players at the professional level in the women's tournament so that we could approve or reject this influence and provide valuable information for the coaches about the players' condition at the day of the match or even weeks of training. This can greatly contribute to success of the players.

Purpose: Despite many researches that have been done in the field of biorhythm and due to the lack of a single view among scholars, as well as the importance of forecasting the performance of athletes to improve their performance, the purpose of this study is to determine the relationship between biorhythm (physical cycle) and sports performance in Iranian Super League women's basketball players.

\section{Material and methods}

Participants: The statistical population was women's basketball players in 1394-95 women's Super League and the statistical sample included all of the players of the teams qualified as semi-finalists (44 players).

Procedure: This study is an applied research and is a descriptive correlational study in terms of nature. The tournament was held in the form of home and international matches. The tool, used in this research, was athletes ' performance questionnaire (Charbonneau, 2001) containing five questions on a Likert scale of 1 (poor) to 5 (excellent) points. This questionnaire was delivered to the coaches after each match so that they could evaluate the players' performance with answering its questions based on the day of the match, comparing to previous matches and other days and their knowledge and expectations of the players. The results of the competition were recorded in which code 1 denoted a win and code 2 denoted a loss.

Biorhythm software and a form to collect information were used in order to determine the athletes ' biorhythm. Data on age and date of birth of the athletes were collected through a demographic information form. Athletes who were in their menstrual cycle, as well as those who were injured or psychologically damaged by 
being faced with some bad news before the match were excluded for integration purpose. The Farsi version of Biorhythm software was employed to draw athletes' biorhythm. The software drew players` biorhythm by entering their date of birth. [21].

After revealing the athletes` biorhythm through Biorhythm software, all of their cycles were examined.

Based on the physical cycle of biorhythm in each course of collecting questionnaire's data, three different phases were distinguished:

1. Cycle is located above the zero line (positive phase).

2. Cycle is located below the zero line (negative phase).

3. Cycle is located in the zero and critical range (48-hour).

Statistical analysis: Inferential statistics such as Pearson correlation coefficient, independent two-sample T-test, Chi-Square test, one-way analysis of Variance (ANOVA) and Tukey post hoc test were used to investigate the hypotheses. It should be noted that all statistical analyses were performed by using SPSS software at the significance level of $(\mathrm{P} \geq 0.05)$.

\section{Results of the researches}

The results given in table 1 show that there is a positive relationship between physical energy and athletes` performance, but this relationship is not significant $(\mathrm{r}=0.02, \mathrm{p}=0.851)$.

Table 1. Relationship between biorhythm (physical cycle) and sport performance

\begin{tabular}{llll}
\hline \multicolumn{1}{c}{ Variable } & N & Pearson Correlation Coefficient & p-value \\
\hline $\begin{array}{l}\text { Relationship between biorhythm (physical cycle) and } \\
\text { sport performance }\end{array}$ & 88 & 0.02 & 0.851 \\
\hline
\end{tabular}

The results of table 2 show that there is no significant difference between the energy average in two groups of win and loss $(\mathrm{p}=0.851)$.

Table 2. Independent T results of mean physical energy with match results

\begin{tabular}{lllllll}
\hline cycle & Groups & N & Mean \pm SD & df & t & p-Value \\
\hline \multirow{2}{*}{ Physical } & Winner & 47 & $51.83 \pm 26.38$ & & & \\
& Loser & 41 & $52.95 \pm 29.48$ & 86 & 0.188 & 0.851 \\
\hline
\end{tabular}

The results of table 3 show that there is no significant differences between athletes' performance in three levels (positive, negative and critical) of physical cycle $(\mathrm{p}=0.96)$.

Table 3. One-way ANOVA results of Athletes' performance with different levels physical cycle

\begin{tabular}{llllll}
\hline & Sum of Squares & df & Mean Squares & F & P-value \\
\hline Between Group & 64.8 & 2 & 32.4 & & \\
Within Group & 59900 & 75 & 798.68 & 0.041 & 0.96 \\
Totla & 59964 & 77 & - & & \\
\hline
\end{tabular}

Based on the results of table 4, the value of Chi-Square is 2.63 showing a significant relationship between different levels of physical cycle and match results (win or loss) $(\mathrm{p}=0.26)$.

Table 4. Relationship between match results with different levels physical cycle

\begin{tabular}{|c|c|c|c|c|}
\hline Physical cycle & & $\begin{array}{l}\text { match } r \\
\text { Winner }\end{array}$ & loser & Total \\
\hline Positive & Frequency & 17 & 24 & 41 \\
\hline
\end{tabular}




\begin{tabular}{lllll}
\hline & percent & 41.5 & 54.5 & 100 \\
Negative & Frequency & 13 & 14 & 27 \\
& percent & 48.1 & 51.9 & 100 \\
critical & Frequency & 7 & 3 & 10 \\
Total & percent & 70 & 30 & 100 \\
Chi-squared test & & 37 & 41 & 78 \\
\hline
\end{tabular}

\section{Discussion}

According to the Biorhythm theory, people face internal changes at different times, which may be the cause of different behaviors and performances at different times. Biorhythm can determine good and bad of people's performance in different days. In fact, according to biorhythm in critical days, people inherently tend to be inaccurate and if they do some risky works at this period, this inaccuracy may cause them some problems. Interpretation of different conditions of biorhythm physical cycle in different situations can be a great help to justify and evaluate physical conditions and performance of athletes. Also according to biorhythm, knowing the physical condition of athletes can help to plan for getting the best results (Shaebani Bahar, 2013). This is the question that needs to be answered here: is there any relationship between biorhythm and performance of athletes?

The results of this study showed that there is no significant relationship between physical energy and performance of athletes. This means that increasing the level of physical energy has no effect on increasing or decreasing the performance of athletes on the day of the match. This is consistent with findings of Reilly et al. (1983) about the impact of the biorhythm on the performance of professional women athletes, and with findings of Wolcott et al. (1977) about professional hammer throw. Also this is consistent with the findings of Connor \& Molly (1991), and Ezanlou et al. (2007) who investigated the biorhythm experimentally and concluded that biorhythm cycle has no effect on people`s performance. Perhaps one of the reasons of these consistencies is the professional level of the studied tournament. However, the results were inconsistent with the findings of the Biorhythm theory by Willis (1972), Wallerstein and Roberts (1973), Wilhelm Felix (1928) and Alfred Teleshter (1920) which all believed that the performance is improved when the biorhythm cycle is located at the top of the baseline. Also this is inconsistent with the findings of Shaebani Bahar et al. (2013). However, these studies evaluated the performance of novice athletes in training and student matches and perhaps the differences in the level of competition and maintaining physical and intellectual fitness of athletes in professional events are the cause of these different results. We can also mention the difference in performance in practices and matches as a reason in this regard. Because an athlete can control many factors in a practice, but the performance in a match will be very different from practice and its conditions. However, we cannot certainly acknowledge the inefficiency of this software, because it has increased the performance on positive days of physical cycle in some cases, such as factories, staff and students. This increase was proved to be significant based on the findings of Rabiei \& KhatamiNo (2011) and Hosseini \& Eshraghi (2010). Also based on the findings of Vazifedoust et al. (2013), the biorhythm has an impact on the quality of managers' decision-making. Perhaps this software works fine when it comes to the people who do not need permanent intellectual, emotional and physical fitness.

The results of the study did not show any significant difference between the performance of athletes at three levels of physical cycle (positive, negative and critical) $(\mathrm{p}=0.96)$. These results are consistent with findings of Jenkins et al. (2011), Lester (1990), Pilianidis et al. (1993), Quigley (1982), Faria and Elliott (1980), and are inconsistent with the findings of Moemeni (2012), Rabiea and KhatamiNo (2011), Hosseini and Mehdi Zadeh (2009), Singh and Sharma (2011), Parikh et al. (2010), Maura (2009) and Taylor (2004). So, it can be concluded that awareness of positive, negative and critical situations of physical cycle is not effective in achieving desirable results for athletes' performance. Therefore, it is not possible to predict the athletes' best time to do the match when their physical cycle is in positive condition. In fact, there are many factors affecting the quality of performance in a match, such as physical condition, having no injuries, good condition of training before the match and getting enough rest and sleep before the match.

With regard to relationship between match results (win or loss) and different conditions of physical cycle, the findings showed that positive and negative conditions of athletes have no effect on their wins or losses. This is inconsistent with the findings of Moldovan (2011) which showed that the performance of athletes is higher in positive conditions of biorhythm cycle. This is also inconsistent with the findings of Ehsani and Parsa (2010), who revealed a decrease in employees ' performance on critical days and an increase on positive days, and findings of Taylor (2004) on the reduction of work-related accidents in the positive phase of the cycle. It can be concluded that the conditions and situations that happen in a match such as key players being sent off, biased referee judgments, unfavorable conditions of safety and security and even bad weather conditions all may affect a team`s 
winning and losing. Therefore, the positive condition of the team is not a reasonable predictor for its winning chance.

With regard to receiving many stimuli by the athletes during the matches and the ability and experience of professional athletes in competition with controlling intellectual and emotional conditions as well as maintaining physical fitness at desirable level for major events, it can be concluded that using biorhythm in sports and for athletes in a major event could be effective in increasing athlete's motivation provided that it is in a positive phase. It should also be noted that the coach should not use this software fraudulently for increasing motivation of the athletes. Otherwise, for professional athletes who need to keep their physical conditions and energy at the best level during matches, the negative phase of this theory could implicate some undesirable intellectual effects during the competitions. However, the coaches can use biorhythm in practices for increasing athletes ' performance, motivation and self-confidence at the best condition of physical cycle, but this software does not cause any increase or decrease in performance for match conditions. Of course, more researches need to be done in this area.

\section{Conclusions}

So any long-term planning to use biorhythm as a basis for competition and training is a bit unwise. Although knowing intellectual features and physical conditions of the athletes could help coaches to select players for important matches, but this software is hardly helpful for the coaches in this case. It should be noted that professionalism requires athletes to maintain their intellectual, emotional and physical fitness to compete in the best conditions. This could be one of the most important factors indicating why Biorhythm theory does not apply to professional athletes.

\section{Conflict of interests}

The authors declare that there is no conflict of interests.

\section{References:}

1. Singh R \& Sharma R. The influence of "biorhythm" on the incidence of injuries among Agra foundry workers. International Journal, 2011;3: 20-25.

2. Raut TS, \&.Kaware SHH. Comparative study of Biorhythms with Various physical fitness components. International Referred Research Journal, 2011;4:32-33.

3. Faria IE, \& Elliott TL. Biorhythm patterns of maximal aerobic power of females. The Journal of sports medicine and physical fitness, 1980; 20(1): 81-86.

4. Ehsani A, Parsa Y. Biorhythm based on the theory of industrial management decision-making process for the implementation of automation in production units. 2nd International conference on industrial Automation. 2011; P. 11-16. (Article in Farsi)

5. Parikh RH, Askhedkar RD, Singh MP. Biorhythms for accident prevention. International Journal of Multidisciplinary Research and Advances in Engineering, 2010; 2(1):217-232.

6. Zareian E, Rabbani V, Saeedi F. The Effect of Physical Biorhythm Cycle on some Physical Fitness Factors of Adolescent Volleyball Players. Annals of Applied Sport Science, 2014; 2(1): 11-20.

7. Ezanloo B, Ebrahimi S, Ghavam Habibi M, Asgarabad. The Investigation of the Validity of Intellectual Cycles of Biorhythm through Cognitive Functions. Research in Psychological Health. 2007;1(3):38-50. (Article in Farsi)

8. Javaherdashti R. The Management of Man's Physiological Behavior. TADBIR. 2001; 11(109):58-60. (Article in Farsi)

9. Hoseini SM, Mehdizadeh A, Ashrafi. Biorhythm and Educational Performance: (Students of Islamic Azad University, Firoozkuh Branch. Pajouheshgar). Journal of Management, 2009;6(13):70-6. (Article in Farsi)

10. Rabiee A, Khatamino F. Study of the Relationship between Biorhythm and Staff's Job Satisfaction (Case study: Nargan Company). Quarterly Management \& Human Resurces in Oil Industry, 2011; 2(8):29-52.

11. Buttery, T.J. The influence of biorhythm on human physical, emotional, and intellectual behavior. Education. 1977; 98(2), 117-121.

12. Sha'bani Bahar GR, Samadi A, Momeni Piri S. The relationship between Intellectual and Emotional cycles with respect to Biorhythm Theory and athletes' sport performance. Journal of Sport Psychology Studies, 2013; 2(3):13- 28. (Article in Farsi)

13. Moemeni Piri S, Shabani Bahar G. The Determination of Relationship between Biorhythm (Physical Cycle) and Sport Performance in Athletes of Individual Sports in Hamadan Province. Master thesis Physical Education, Bu-Ali Sina University of Hamedan; 2012.

14. Willey P \& Farnkoni A. Scientific tests of biorhythmology do not support its claims. In: Frazier (ED.) Science confronts the paranormal. Buffalo, NY: Prometheus; 2003. 219-221.

15. Dehghan AM. The psychological factors affecting investment in stock. A Monthly Magazine TADBIR on Management. 2008;196: 60-63. (Article in Farsi) 
16. Maura S. Bio-periodicity and its influence on the football results obtained by the student teams during the "university spring" sport competition. Citius Altius Fortius; 2009.

17. Moldovan E, enoiu RS, ruxanda RA, \& leibovici A. The influence of the human biorhythm in the performance sport activity. Gymnasium, 2011; 12: 34-46.

18. Talor CG. The structure \& dynamics of the biorhythm, collected works, 8, R.F.C hull, (trans.). Bollingen series XX, pantheon books; 2004.

19. Zollitsch HG. Biorhythms and management. Industrial management, 1976;11: 1- 4.

20. Reilly T, Young K \& Seddon R. Investigation of biorhythms in female athletic performance. Applied Ergonomics, 1983; 14(3), 215-217.

21. Bio-chart. Biorhythms -Some History. Available from: http://www.bio-chart.com/geschichte.html. 2014 (accessed 22.05.2016).

22. Joncas SX, Carrier N, Nguyen M, \& Farand P. Biorhythm Theory Does Not Predict Admission for Acute Myocardial Infarction. The Journal of Alternative and Complementary Medicine. 2011; 17(2): 143-146.

Information about the authors:

Shafiee Shahram; Assistant professor; http://orcid.org/00000002-8208-0846; shafieeshahram@gmail.com; Faculty of Physical Education and Sport Sciences, University of Guilan; Box 1841, Rasht, Iran.

Rahim Ramezaninezhad; professor; http://orcid.org/00000003-4646-1892; rramguil@yahoo.com; Faculty of Physical Education and Sport Sciences, University of Guilan; Box 1841, Rasht, Iran.

Hakime Afrouzeh; MA of sport management; http://orcid.org/0000-0002-4774-5101;

haafroozeh@yahoo.com; University of Guilan; Box 1841, Rasht, Iran.

Vahid Rabbani; MA of sport management; http://orcid.org/0000-0001-8302-5251;

vahid.rabbani66@gmail.com; Allameh Tabataba'i University, P.O. Box 14155-8473 Tehran, Iran.

Cite this article as: Shafiee Shahram, Rahim Ramezaninezhad, Hakime Afrouzeh, Vahid Rabbani. The relationship between biorhythm (physical cycle) and sports performance in women's basketball. Physical education of students, 2016;3:58-64. doi:10.15561/20755279.2016.0308

The electronic version of this article is the complete one and can be found online at: http://www.sportpedu.org.ua/html/arhive-e.html

This is an Open Access article distributed under the terms of the Creative Commons Attribution License, which permits unrestricted use, distribution, and reproduction in any medium, provided the original work is properly cited (http://creativecommons.org/licenses/by/4.0/deed.en).

Received: 18.06 .2016

Accepted: 26.06.2016; Published: 28.06.2016 